\title{
Monte Carlo Simulation of the Rapid Crystallization of Bismuth-Doped Silicon
}

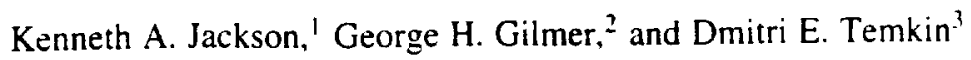 \\ 'Materials Science and Engineering. University of Arizona, Tucson. Arizona 857/2 \\ ${ }^{2}$ AT\&T Bell Laboratories, Murray Hill, New Jersey 07974 \\ ${ }^{3}$ Central Scientific-Research Institute of Ferrous Metallurgy: Moscow 107005. Russia \\ (Received 17 January 1995)
}

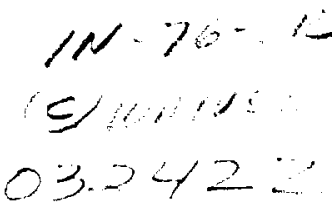

In this Letter we report Ising model simulations of the growth of alloys which predict quite different behavior near and far from equilibrium. Our simulations reproduce the phenomenon which has been termed "solute trappirty," where concentrations of solute, which are far in excess of the equilibrium concentrations, are observed in the crystal after rapid crystallization. This phenomenon plays an important role in many processes which involve first order phase changes which take place under conditions far from equilibrium. The underlying physical basis for it has not been understood, but these Monte Carlo simulations provide a powerful means for investigating it.

PACS numbers: $61.50 . \mathrm{Cj}, 61.46 .+\mathrm{w}, 64.60 .-\mathrm{i}$

Rapid cooling is being used increasingly to produce fine, tailored microstructures, to produce fine dispersions by rapid quenching of small particles, and to modify the surface properties and structure of materials by rapid thermal processing or by laser irradiation. In some materials, the amorphous structure of the liquid is preserved in the form of a metastable glassy phase [1-3]. In other materials, equilibrium phases are suppressed, and unstable or metastable phases, which do not exist otherwise, form during a rapid quench. In most materials, compositions outside the equilibrium range can be obtained. Related nonequilibrium segregation effects such as the "facet effect" in the Czochralski growth of silicon [4,5] are present at relatively slow growth rates. There is no sound physical understanding or theoretical framework for these and many other nonequilibrium effects associated with first order phase changes which take place far from equilibrium.

The best data available for the formation of phases which have nonequilibrium compositions have been obtained for the incorporation of dopants into silicon during very rapid solidification following laser melting of a surface layer [6-14]. These dopants are incorporated into the crystal at high concentrations which are metastable at any temperature, and they will precipitate during subsequent annealing. The quantitative measure of this effect is the distribution coefficient, also known as the $k$ value, which is the ratio of the concentration of dopant in the solid at the interface to the concentration of dopant in the liquid at the interface. The $k$ value increases dramatically with growth rate in these experiments, from an equilibrium value of 0.001 to a value approaching 1 . Several models have been proposed to account for the phenomenon [15-18], and the model which has been compared most extensively with experiment is due to Aziz [17-21]. The underlying physical basis for this model is controversial, but it does provide a distribution coefficient which can differ significantly from the equilibrium distribution coefficient, and it has been used to fit to experimental results using reasonable values for the diffusion coefficient.

Previous Monte Carlo simulations, based on the Ising model applied to alloys, have not reproduced these experimental results [22]. Why this is so has been a mystery, since our present understanding of the atomic scale processes involved in crystallization is based on the Ising model, and Monte Carlo simulations [23-26] guided the development of this understanding, which includes the surface roughening transition and its central role in the equilibrium properties of surfaces and interfaces, and in the kinetics of interface motion during crystal growth [27].

The simulations reported here have been done in two dimensions and are a special version of a two-dimensional random walk. In order to simulate crystal growth in two dimensions, the position of the "walker" becomes a line which represents an interface. In three dimensions, a surface represents the interface. The crystal is on one side of this line or surface, and the liquid is on the other. The probability that the interface jumps backward or forward at a site depends on how many of its nearest neighbors are on the crystal side or on the liquid side of the interface. This lateral coupling tends to keep the interface planar, and so effectively introduces a surface tension. The probability of interface jumps in the two directions can be biased to simulate nonequilibrium melting or freezing. To model an alloy, special sites are introduced to represent the alloying element or dopant. There is a different bias for interface jumps across these sites than at the normal sites. The simplest case conceptually is when these special sites are stationary. This corresponds to diffusionless growth, which is the limiting case for the diffusion of atoms being slow compared to the rate at which the interface moves. In this limit, the liquid and solid both have the same composition since only the interface moves, not the atoms, and so the $k$ value is 1. The net rate of motion of the interface depends on an average of the biases for the two types of sites. The 
special sites can also be made to move around in order to simulate diffusion. In most alloys, diffusion in the liquid is very rapid compared to diffusion in the solid, and so it is usual to assume that there is diffusion only in the liquid. The usual near-equilibrium growth conditions for alloys are reproduced when the special sites move around rapidly compared to the rate at which the interface moves. The "equilibrium" $k$ value in this case depends on the relative biasing for the two types of sites. When the biasing is adjusted so that the equilibrium $k$ value is less than 1, the dopant atoms tend to stay in the liquid and diffuse away from the interface. But, as pointed out above, the $k$ value is 1 if the special sites are stationary, independent of the biasing. There is a transition between these two extremes which occurs when the jump rate for the special sites is comparable to the rate at which the interface moves. We believe this to be the origin of solute trapping behavior, and it is evident that this effect should be present in this model as well as in experiment.

Simulations were reported earlier $[28,29]$ using this model for "diffusionless" transformations for which the $k$ value is 1 . These provided the first clear-cut confirmation of the theoretically expected behavior for a diffusionless transformation, that is, that "freezing" or "melting" should be reversible for temperatures above and below $T_{0}$, the (composition dependent) temperature at which the free energy of a solid alloy is equal to the free energy of a liquid alloy with the same composition. This is a significant result because the jump rates of the interface were specified in terms of the chemical potentials of the atoms, not in terms of the free energies of the phases. This suggests that the simulations contain the correct physical model to explain solute trapping. These simulations also provided the first information about crystallization kinetics in diffusionless transformation.

The Monte Carlo simulations reported here are in the transition region where the $k$ value is intermediate between the equilibrium value and 1 , and the diffusion coefficient and the growth temperature in the simulations have been adjusted to reproduce the experimentally measured distribution of a dopant in a silicon crystal after rapid recrystallization of a surface layer. The transition rates at the interface for the Monte Carlo scheme used here are similar to those used previously $[22,28,29]$. The transition probability for an atom to leave the crystal to join the liquid can be written as

$$
P_{k}^{-}=P_{k}^{0} \exp \left[\sum\left(-\frac{\phi_{k l}^{C_{j}}}{k_{B} T}\right)\right] .
$$

Here $P_{k}^{0}$ is a constant, $k_{B}$ is Boltzmann's constant, and $T$ is the temperature. The superscript $C$ identifies the atom as being in the crystal, and the subscript $k$ identifies the species of the atom. The summation is over the nearest neighbors of the atom; each nearest neighbor is identified by a pair of indices $j$ and $l$, where $j$ is either $C$ (crystal) or $L$ (liquid), and $l$ identifies the species of the neighboring atom. $\phi_{h l}^{i i}$ is the energy of the bond between one atom defined by $i k$ and its neighbor defined by $j l$. Similarly, the probability of an atom going from liquid to crystal is

$$
P_{k}^{+}=P_{k}^{0} \exp \left(-\frac{\Delta S_{k}}{k_{B}}\right) \exp \left[\sum\left(-\frac{\phi_{k l}^{L j}}{k_{B} T}\right)\right],
$$

where $\Delta S_{k}$ is the entropy of fusion for the species $k$. This form preserves microscopic reversibility. The simulations reported here start with both solid atoms and liquid atoms present in a two-dimensional array with an interface between them. Diffusion in the liquid is modeled by the interchange of two adjacent atoms of different species, with a probability which is chosen so that the average jump rate $\Gamma$ is some multiple (or fraction) of $P_{k}^{0}$. A temperature is chosen, and the transition probabilities $P_{k}$ are calculated from Eqs. (1) and (2). Individual atoms at the interface join or leave the crystal based on these transition probabilities. A normalized rate of motion of the interface is then calculated from the net motion of the interface.

Figure 1 shows typical experimental data for silicon implanted with bismuth [10]. The as-implanted distribution of bismuth is shown as the dashed line. The sample was surface melted with a laser to a depth of about $2 \mu \mathrm{m}$. The interface layer stayed molten for about $2 \mu \mathrm{s}$, during which time the implanted atoms diffused in the liquid, spreading out the as-implanted distribution. The interface then came back toward the surface, pushing the bismuth atoms ahead of it, resulting in the distribution determined by Rutherford backscattering as shown by the open circles. An experimental distribution coefficient ( $k$ value) was determined [10] by estimating the amount by

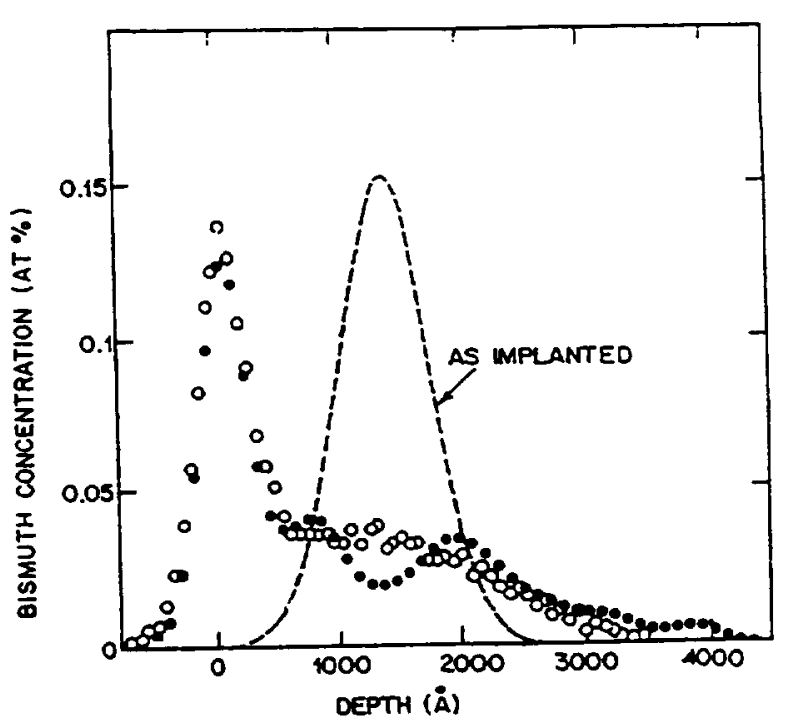

FIG. 1. The open circles are the experimental data for the depth distribution of bismuth implanted into silicon after laser melting of a surface layer. The closed circles are the depth distribution of bismuth atoms from the Monte Carlo simulations. 
which the implanted bismuth distribution spread out by diffusion while the surface layer was liquid, then adjusting the $k$ value to fit the final bismuth distribution which resulted from the passage of the interface. Although the equilibrium $k$ value for bismuth in silicon is $8 \times 10^{-4}$, the data were fitted with a $k$ value of 0.1 . This experiment, and many other similar experiments, unequivocally established that the $k$ value is strongly growth rate dependent.

Simulations were performed for dilute $\mathrm{Si}: \mathrm{Bi}$ alloys by using bond energies, which werè calculated from the thermodynamic properties of the alloy using a regular

\begin{tabular}{cccccccc}
$T_{m}^{\mathrm{Si}}$ & $T_{m}^{\mathrm{Bi}}$ & $\Delta S_{\mathrm{Si}} / k_{B}$ & $\Delta S_{\mathrm{Bi}} / k_{B}$ & $k_{e}$ & $\left(\phi_{\mathrm{SiSi}}^{C C}-\phi_{S i S i}^{L L}\right) / k_{B}$ & $\left(\phi_{B i B i}^{C C}-\phi_{B_{B} B_{i}}^{L L}\right) / k_{B}$ & $\left(\phi_{\mathrm{SiBi}}^{C C}-\phi_{S i B i}^{L L}\right) / k_{B}$ \\
\hline $1688 \mathrm{~K}$ & $544.5 \mathrm{~K}$ & 10 & 10 & $8 \times 10^{-4}$ & $8440 \mathrm{~K}$ & $2722.5 \mathrm{~K}$ & $5430.8 \mathrm{~K}$
\end{tabular}

$T_{m}^{\mathrm{Si}}$ and $T_{m}^{\mathrm{Bi}}$ are the melting points of silicon and bismuth, respectively.

A two-dimensional array of $150 \times 1500$ atoms was used, which corresponds to a sample that is about $45 \mathrm{~nm}$ wide by $450 \mathrm{~nm}$ deep. This depth is about the same as the total penetration depth of bismuth atoms in the experiment. Since the as-implanted profile spreads out due to diffusion in the liquid during the time that a liquid layer exists, a profile for the distribution of bismuth atoms at the time when the crystallization front reached it was established as follows. 63 bismuth atoms were inserted into a layer $140 \mathrm{~nm}$ deep in the crystal, which corresponds to the total implant concentration and to the average implant depth. These atoms were then allowed to diffuse to a half-width of the order of $100 \mathrm{~nm}$, comparable to the width of the bismuth distribution in the experiment just before the recrystallization front reached the deepest bismuth atoms. The same distribution could have been achieved by letting the atoms diffuse while the interface penetrated into the crystal and then returned to the depth of the bismuth atoms, but the crystallization front does not interact with the bismuth atoms during this period. The actual $\mathrm{ms}$ half-width of the distribution used was 340 atomic layers. The closed circles in the figure show the distribution of the bismuth atoms in the simulations after the passage of the crystallization front at a temperature of $1400 \mathrm{~K}$, with the diffusion jump rate 40 times the arrival rate of atoms at a repeatable step site. The final positions of the atoms were smoothed by using Gaussian averaging to simulate the experimental broadening associated with the Rutherford backscattering depth measurement. The wiggles in the final distribution results from having a total of only 63 bismuth atoms in the simulation. The simulations reproduce the experimental result, giving a $k$ value of 0.1 for these growth conditions. Similar agreement has been obtained for data in Ref [13]. Simulations run at other temperatures (growth rates) and with various liquid diffusion coefficients indicate that the $k$ value depends on solution model for the solid and an ideal solution model for the liquid. with an equilibrium $k$ value of $8 \times 10^{-4}$. The entropy of fusion, which determines the roughness of the interface at equilibrium, was chosen to stay below the two-dimensional critical point, and to make the repeatable step density, which depends on the roughness of the interface. similar to that for silicon. A repeatable step site (also known as a kink site) is an interface site which has half of its nearest neighbor sites occupied by atoms of the crystal. The same values were used for the $C L$ bonds and for the $L L$ bonds. The values used in these simulations are listed here:

both. Similar results have been obtained with simulations in three dimensions.

In the previous Monte Carlo simulations [22], atoms arrived randomly at surface sites, as given by Eq. (2), and departed from surface sites as given by Eq. (1). The $k$ value was taken as the ratio of the concentration of dopant in the growing crystal to the concentration of dopant in the arriving atoms. The arrival and departure rates from the crystal were identical to those used here, but there was no liquid phase present. This was believed to be a valid model since dopant incorporation into a crystal, including solute trapping, is a process which takes place at the interface and provides the boundary condition at the interface for the diffusion field in the liquid. This implies that the diffusion process in the liquid can be separated from what happens at the interface. However, in these simulations [22] it was found that the $k$ value did not increase very much from the equilibrium value with growth rate. Agreement with experimental data could be obtained only by introducing surface segregation and stress near the interface. The results presented here indicate that the essential difference between the two simulation schemes is the presence of the liquid phase.

The Ising model Monte Carlo computer simulations reported here reproduce experimental results on solute trapping with the only input being equilibrium thermodynamic data, a diffusion coefficient for the atoms in the liquid phase, and a growth temperature. This model is actually the simplest possible scheme for simulating alloy crystallization, but it exhibits unexpected and quite complex behavior. We plan to explore this behavior in order to develop an understanding of the implications of the simple underlying assumptions. These simulations provide a powerful and flexible means of exploring the consequences of these simple assumptions for crystal growth, and it is expected that this will provide significant new insights into the crystallization of multicomponent materials. 
The part of this work which was carried out at the University of Arizona was supported by NASA Contract No. NAG8-944.

[1] P. Duwez, ASM Trans. Q. 60.606 (1967).

[2] P. Duwez, Prog. Solid State Chem. 3. 377 (1967).

[3] Ultrarapid Quenching of Liquid Alloys, edited by H. Herman (Academic Press, New York, 1981).

[4] T. Abe, J. Cryst. Growth 24/25, 463 (1974).

[5] A.J.R. de Kock, in Crystal Growth and Materials, edited by E. Kaldis (North-Hohand. Amsterdam, 1977), p. 693.

[6] P. Baeri, J. M. Poate, S. U. Campisano, G. Foti, E. Rimini, and A. G. Cullis, Appl. Phys. Lett. 37, 912 (1980).

[7] P. Baeri, G. Foti, J. M. Poate, S. U. Campisano, and A. G. Cullis, Appl. Phys. Lett. 38, 800 (1981).

[8] C.W. White, S. R. Wilson, B. R. Appleton, and F.W. Young Jr., J. Appl. Phys. 51, 738 (1980).

[9] C. W. White, B. R. Appleton, B. Stritzker, D. M. Zehner, and S.R. Wilson, Mater. Res. Soc. Symp. Proc. 1, 59 (1981).

[10] P. Baeri, G. Foti, J. M. Poate, S. U. Campisano, E. Rimini, and A. G. Cullis, Mater. Res. Soc. Symp. Proc. 1, 67 (1981).

[11] C. W. White, D. M. Zehner, S. U. Campisano, and A. G. Cullis, Surface Modification and Alloying (Plenum, New York, 1983), p. 81.

[12] M. J. Aziz, J. Y. Tsao, M. O. Thompson, P. S. Peercy, C. W. White, and W.H. Christie, Mater. Res. Soc. Symp. Proc. 35, 153 (1985).

[13] M. J. Aziz, J. Y. Tsao, M. O. Thompson, P. S. Peercy, and C. W. White, Phys. Rev. Lett. 56, 2489 (1986).
[14] M.J. Aziz and C.W. White. Phys. Rev. Lett. 57. 2675 (1986).

[15] K.A. Jackson. G. H. Gilmer. and H.J. Leamy, in Laser and Electron Beam Processing of Materials, edited by C.W. White and P.S. Peercy (Academic. New York. 1980), p. 104.

[16] R. F. Wood. Appl. Phys. Lett. 37. 302 (1980).

[17] M. J. Aziz, J. Appl. Phys. 53, 1158 (1982).

[18] M. J. Aziz, Appl. Phys. Lett. 43. 552 (1983).

[19] M. J. Aziz. Mater. Res. Soc. Symp. Proc. 23. 369 (1984).

[20] L. M. Goldman and M.J. Aziz. J. Mater. Res. 2, 524 (1987).

[21] M. J. Aziz and T. Kaplan, Acta Metall. 36. 2335 (1988).

[22] G. H. Gilmer, Mater. Res. Soc. Symp. Proc. 13, 249 (1983); Mater. Sci. Eng. 65. 15 (1984).

[23] H. J. Leamy and K. A. Jackson. J. Appl. Phys. 42, 2121 (1971).

[24] H.J. Leamy and G. H. Gilmer. J. Cryst. Growth 24/25. 499 (1974).

[25] H. J. Leamy, G. H. Gilmer, and K. A. Jackson. Surface Physics of Materials, edited by J.M. Blakeley (Academic. New York, 1975), p. 319.

[26] G. H. Gilmer and K. A. Jackson, 1976 Cnystal Growth and Materials, edited by E. Kaldis and H.J. Scheel (NorthHolland. Amsterdam, 1977), p. 80.

[27] J.D. Weeks and G. H. Gilmer, Adv. Chem. Phys. 40, 157 (1979).

[28] K. A. Jackson, G. H. Gilmer, D. E. Temkin, J. D. Weinberg. and K. Beatty, J. Cryst. Growth 128, 127 (1993).

[29] K. A. Jackson, G.H. Gilmer, D.E. Temkin, and $\mathrm{K}$. Beatty, in Proceedings of the East-West Surface Science Workshop '94 (to be published). 